\title{
Leaking splenic artery pseudoaneurysm - an uncommon complication of pancreatitis
}

\author{
R.M.T.M Gunawardena ${ }^{1}$, S.S Wanigasooriya ${ }^{1}$, M.A.E Perera ${ }^{1}$, P.A.Y.P Weerawardhana ${ }^{2}$ \\ 1 Base hospital Awissawella, Sri Lanka. \\ 2 Base hospital Warakapola, Sri Lanka.
}

Key words: Splenic artery pseudoaneurysm; pancreatitis complications

\section{Introduction}

Pseudoaneurysm formation of the splenic artery is a rare but potentially life threatening complication of pancreatitis [1]. We present a case of a 43 year old male with acute on chronic pancreatitis complicated with a leaking pseudoaneurysm of the splenic artery.

\section{Case presentation}

A 43 year old male patient was admitted to the surgical casualty unit of the Awissawella Base Hospital complaining of progressively worsening generalized abdominal pain for two days duration. He has had two similar episodes of pain of a lesser severity within the last eight months. The pain was associated with nausea, vomiting and dizziness. There was no history of fever, hematemesis, melaena or other bleeding manifestations. He was a non-smoker and was not on any long term medication, but has been consuming half a bottle of alcohol daily for the last 5 years.

On examination, he was hypovolemic with a low volume pulse, tachycardia (96bpm) and a low blood pressure of 85/65 $\mathrm{mmHg}$ was detected. The abdomen was distended with diffuse tenderness and guarding upon palpation. His blood investigations revealed a haemoglobin level of $12.7 \mathrm{~g} / \mathrm{dL}$ with normal white cell and platelet counts. Serum creatinine and electrolytes were within normal limits but the amylase level was significantly elevated(1118U/L, normal 20-80).

Subsequent to resuscitation with intravenous fluid, an urgent ultrasound scan of the abdomen was performed. A lesion was noted at the splenic hilum measuring $4.5 \times 3.5 \times 3.5 \mathrm{~cm}$ with a hypo echoic centre containing high velocity colour Doppler signals of arterial type. The lesion was surrounded by an area of mixed echogenicity which was keeping with ultrasonic features of a haematoma. There was significant amount of

Correspondence: R.M.T.M Gunawardena

E-mail: thilinamg@gmail.com

Received: 29-06-2017 Accepted: 02-10-2017

(iD) http://orcid.org/0000-0002-3867-2780 DOI: http://doi.org/10.4038/sljs.v35i3.8411 free fluid noted in the abdomen. The conclusion was a leaking splenic artery pseudoaneurysm with haemoperitoneum.

The patient underwent an emergency laparotomy where a haemoperitoneum of about $1.5 \mathrm{~L}$ was noted on entering the abdominal cavity. The pancreas had a hard consistency with foci of calcifications, which was suggestive of chronic pancreatitis. A mass was found in relation to the tail of the pancreas and the splenic hilum. The splenic artery was ligated and a splenectomy was performed along with distal pancreatectomy. The abdomen was closed with a drain in the lesser sac. Intraoperatively 2 units of packed red cells were transfused. Postoperatively he was managed in the ward and his recovery was uneventful. The abdominal drain was removed on post op day 3 and he was discharged on post op day 5. On discharge his serum amylase has returned to normal levels. Pneumococcal and Haemophilus influenza type B (HiB) vaccines were scheduled to be administered ten days after the discharge from hospital.

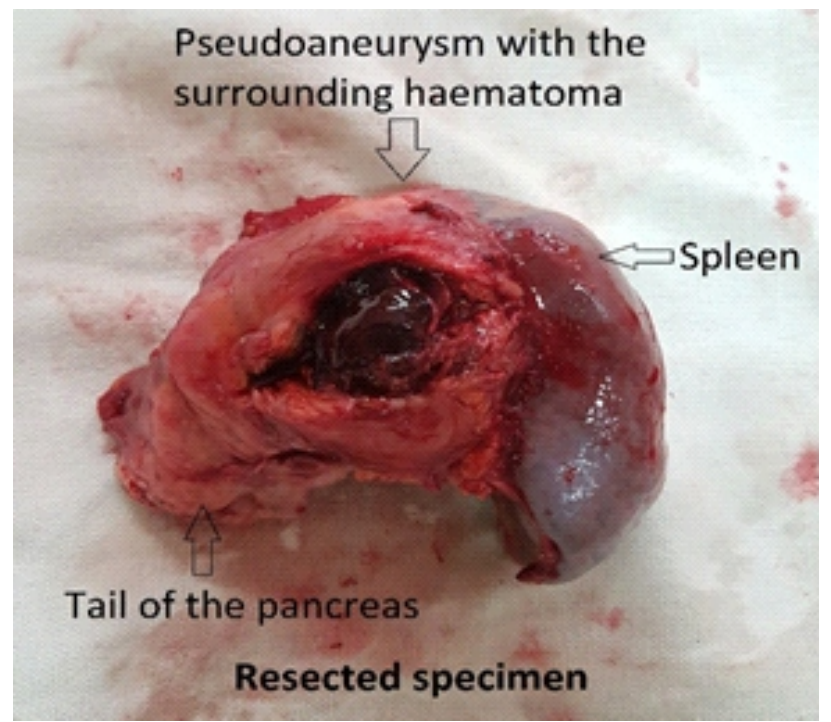

\section{Discussion}

Pseudoaneurysm formation occurs as a complication of pancreatitis in $3.5-10 \%$ [1]. The splenic artery is the most commonly involved vessel accounting for $40 \%$ of cases with gastroduodenal $(30 \%)$, pancreaticoduodenal $(20 \%)$, gastric $(5 \%)$ and hepatic $(2 \%)$ arteries being affected in decreasing

The Sri Lanka Journal of Surgery 2017; 35(3): 5-6 
frequencies [1]. Reported rate of rupture is as high as $37 \%$ in those with a splenic artery pseudo aneurysm, with up to $7.5 \%$ experiencing exsanguinating haemorrhage $[1,2]$.

The pathogenesis of this condition is thought to be due to the digestion of the vessel wall by the proteolytic enzymes released due to the inflammation of the pancreas. Erosion of the vessel by the enzyme rich fluid within a pseudocyst or direct pressure on the vessel by a pseudocyst causing ischaemic damage to the vessel wall are other possible mechanisms [2]. In a review by Tessier et al. it was reported that out of 82 patients with splenic artery pseudoaneurysms caused by pancreatitis, 64 (78\%) had associated pseudocysts [3].

Patients with a ruptured pseudoaneurysm of the splenic artery can present with sudden severe abdominal pain, upper gastrointestinal bleeding with hematemesis, hematochezia, melaena, bleeding from drains left following pancreatic necrosectomy or sudden collapse and death $[1,4]$. Rupture of a pseudoaneurysm can occur in to the general peritoneal cavity, retroperitoneum, in to a pseudocyst, or in to adjacent hollow viscera. Rupture in to the pancreatic duct with bleeding is a rare occurrence, and is known as hemosuccus pancreaticus [4].Angiography is considered the gold standard for imaging of this condition [2]. However contrast enhanced CT and ultrasound with colour Doppler are viable alternatives [3]. In a resource limited environment as this, ultrasound which is cost effective and does not require contrast material is extremely useful and colour Doppler may demonstrate flow within the pseudoaneurysm, as in our patient [3].

Size of the lesion is not a predictor of the risk of rupture hence intervention is recommended for all with this condition [3]. The patient who is haemodynamically unstable due to bleeding splenic artery pseudoaneurysm requires immediate surgery with resuscitation [1]. Splenectomy with or without distal pancreatectomy is the most fail safe procedure described with no reported failures [3]. Splenectomy is associated with increased susceptibility to serious infection and distal pancreatectomy carries the risk of pancreatic duct leaks and fistula formation. Vaccination with pneumococcal, $\mathrm{HiB}$, meningococcal and yearly influenza vaccines are recommended for post splenectomy patients and some patients with a high risk of pneumococcal infection are started on lifelong antibiotic prophylaxis following splenectomy [5].
Ligation of the splenic artery alone with splenic preservation is an option in some patents but it is associated with a recurrence rate of up to $43 \%$ [3]. In the elective setting, open surgery for splenic artery pseudoaneurysms is associated with a $0.5 \%$ risk of operative mortality [2].In the stable patient, angiography with trans catheter embolization is an option with lesser morbidity and mortality with success rates up to $85 \%$ [2]. Post procedure pain, peripancreatic abscesses, splenic infarctions and abscesses, and death are reported complications following embolization of the splenic artery [3].

\section{Conclusion}

In a haemodynamically unstable patient with pancreatitis, the possibility of a leaking peripancreatic pseudoaneurysm should be suspected although it is an uncommon complication. Ultrasound scan with Doppler can accurately diagnose this condition and is most suited for a resource limited setting. Laparotomy with splenectomy and distal pancreatectomy is the commonly preferred procedure in a haemodynamically unstable patient although transcatheter embolization may be preferred in a stable patient at a centre with expertise.

\section{References}

1. Mallick IH, Winslet MC. Vascular Complications of Pancreatitis. JOP. J Pancreas (Online) 2004; 5(5):328-337. Available from: http://www.joplink.net/prev/200409/02.html

2. Agrawal GA, Johnson PT, Fishman EK. Splenic artery aneurysms and pseudoaneurysms: clinical distinctions and CT appearances. AJR Am J Roentgenol. 2007 Apr;188(4):992-9. Available from:

http://www.ajronline.org/doi/full/10.2214/AJR.06.0794

3. Tessier DJ et al. Clinical features and management of splenic artery pseudoaneurysm: case series and cumulative review of literature. J Vasc Surg. 2003 Nov;38(5):969-74. Available from: http://www.jvascsurg.org/article/S07415214(03)00710-9/fulltext

4. Aoun E, Papachristou GI, Whitcomb D, Ahmad I, Slivka A. Acute deterioration of a woman following acute pancreatitis with pseudocysts. Nat Clin Pract Gastroenterol Hepatol. 2005 Nov;2(11):545-9; quiz 550.

DOI: https://doi.org/10.1038/ncpgasthep0317

5. Davies JM et al. Review of guidelines for the prevention and treatment of infection in patients with an absent or dysfunctional spleen: prepared on behalf of the British Committee for Standards in Haematology by a working party of the Haemato-Oncology task force. Br J Haematol. 2011 Nov;155(3):308-17. DOI: http://doi.org/10.4038/sljs.v34i3.8286

\section{Learning Points:}

- Visceral artery pseudoaneurysm formation is an uncommon but potentially life threatening complication of pancreatitis.

- Splenic artery is the most commonly involved artery in this condition.

- Open surgery is required for the haemodynamically unstable patient with a bleeding splenic artery pseudoaneurysm while endovascular methods are more suited for stable patients. 